\title{
ON A MEAN-Generalized SEMIVARIANCE APPROACH TO DETERMINING \\ THE Hedge Ratio
}

\author{
SHENG-SYAN CHEN \\ CHENG-FEW LEE* \\ KESHAB SHRESTHA
}

\begin{abstract}
A new mean-risk hedge ratio based on the concept of generalized semivariance (GSV) is proposed. The proposed mean-GSV (M-GSV) hedge ratio is consistent with the GSV-based risk-return model developed by Fishburn (1977), Bawa (1975, 1978), and Harlow and Rao (1989). The M-GSV hedge ratio can also be considered an extension of the GSVminimizing hedge ratio considered by De Jong, De Roon, and Veld (1997) and Lien and Tse $(1998,2000)$. The M-GSV hedge ratio is estimated for Standard \& Poor's (S\&P) 500 futures and compared to six other widely used hedge ratios. Because all the hedge ratios considered are known to converge to the minimum-variance (Johnson) hedge ratio under joint
\end{abstract}

\footnotetext{
The authors thank two anonymous referees, the editor, Robert Webb, Gautam Vora, and seminar participants at the 1998 FMA meetings for helpful comments and suggestions. Any remaining errors are the authors'.

*Correspondence author, Faculty of Management, Rutgers University, Janice H. Levin Building, Rockafeller Road, Piscataway, New Jersey 08854-8054; e-mail: lee@business.rutgers.edu
}

Received December 1999; Accepted October 2000

- Sheng-Syan Chen is an Associate Professor in the Department of Finance in the College of Management at Yuan Ze University in Taoyuan, Taiwan.

- Cheng-Few Lee is a Distinguished Professor in the Department of Finance in the Faculty of Management at Rutgers University in New Brunswick, New Jersey.

- Keshab Shrestha is an Associate Professor in the Faculty of Administration at the University of Regina in Regina, Saskatchewan, Canada. 
normality and martingale conditions, tests for normality and martingale conditions are carried out. The empirical results indicate that the joint normality and martingale hypotheses do not hold for the S\&P 500 futures. The M-GSV hedge ratio varies less than the GSV hedge ratio for low and relevant levels of risk aversion. Furthermore, the M-GSV hedge ratio converges to a value different from the values of the other hedge ratios for higher values of risk aversion. (C) 2001 John Wiley \& Sons, Inc. Jrl Fut Mark 21: 581-598, 2001

\section{INTRODUCTION}

There is a considerable body of literature on the determination of optimal hedge ratios based on different criteria. Essentially, this literature suggests that hedge ratios can be classified as either risk-minimizing or utility-maximizing. The risk-minimizing hedge ratios are obtained by a specific risk measure being minimized. Various risk measures are currently being used, such as variance (see Ederington, 1979; Johnson, 1960; Myers \& Thompson, 1989), the mean-Gini coefficient (see Cheung, Kwan, \& Yip, 1990; Kolb \& Okunev, 1992; Lien \& Luo, 1993; Shalit, 1995), and generalized semivariance (GSV), also known as the lower partial moment (see De Jong, De Roon, \& Veld, 1997; Lien \& Tse, 1998, 2000).

The mean-risk hedge ratios, however, are obtained by the maximization of the utility function or a specific function of expected return and risk (see Cecchetti, Cumby, \& Figlewski, 1988; Howard \& D'Antonio, 1984; Hsin, Kuo, \& Lee, 1994; Kolb \& Okunev, 1993). As with the riskminimizing hedge ratio, variance and the mean-Gini coefficient have been used as risk measures in the derivation of the mean-risk hedge ratio. However, the GSV has not been used to represent the risk in the context of the mean-risk hedge ratio.

In this article, we suggest the use of GSV to represent risk in the derivation of the mean-risk hedge ratio. This hedge ratio is called the mean-GSV (M-GSV) hedge ratio. The M-GSV hedge ratio can be considered an extension of the risk-minimizing hedge ratio (called the GSV hedge ratio here) considered by De Jong et al. (1997) and Lien and Tse (1998, 2000). Two important features make the GSV a suitable alternative to other measures of risk. First, the GSV, because of its emphasis on return below target return, is consistent with the risk perceived by managers (see Crum, Laughhunn, \& Payne, 1981; Lien \& Tse, 2000). Second, the GSV is consistent with the concept of stochastic dominance (see Bawa, 1975, 1978; Fishburn, 1977). 
Even though the concept of GSV is consistent with the concept of stochastic dominance, the GSV hedge ratio considered by De Jong et al. (1997) and Lien and Tse (1998, 2000) may not be consistent with stochastic dominance. For the GSV hedge ratio to be consistent with stochastic dominance, it should be independent of the target return. Because the GSV hedge ratios are usually different for different target returns, the independence condition is not satisfied. Therefore, the GSV hedge ratio is not consistent with the concept of stochastic dominance. As an alternative to the GSV hedge ratio, we propose the M-GSV hedge ratio, which is consistent with the risk-return model developed by Fishburn (1977) and Bawa $(1975,1978)$ and used by Harlow and Rao (1989) in the context of asset pricing.

This article differs from the existing literature, especially De Jong et al. (1997) and Lien and Tse (1998, 2000), in many respects. First, as mentioned previously, we propose a GSV-based mean-risk hedge ratio that is consistent with the risk-return model developed by Fishburn (1977) and Bawa (1975, 1978). Because the GSV hedge ratio considered by De Jong et al. and Lien and Tse $(1998,2000)$ ignores the expected return on the hedged portfolio, the M-GSV hedge ratio can be considered an extension of the GSV hedge ratio. Second, we clarify an issue regarding the consistency of the GSV hedge ratio with respect to stochastic dominance. We show that the GSV hedge ratio, considered by De Jong et al. and Lien and Tse (1998, 2000), is not consistent with stochastic dominance. Third, we derive a condition under which the M-GSV hedge ratio will be the same as the minimum-variance (MV) hedge ratio. This condition is different from the normality and martingale conditions required for the convergence of the GSV hedge ratio to the MV hedge ratio. Fourth, we empirically compare the M-GSV hedge ratio with the mean-Gini coefficient hedge ratio, the Sharpe ratio-based hedge ratio, the mean-variance hedge ratio, and the MV hedge ratio. Fifth, we perform the normality and zero expected return (martingale) tests. It is well known that if the spot and futures returns are jointly normal and if the futures price follows a pure martingale process, all the hedge ratios considered will converge to the MV hedge ratio. Therefore, these tests are important from an empirical point of view. Finally, we estimate the semivariance-based hedge ratio for the Standard \& Poor's (S\&P) 500 futures, which is different from the currency futures used by De Jong et al. and the Nikkei 225 futures analyzed by Lien and Tse $(1998,2000){ }^{1}$

${ }^{1}$ Even though both the Nikkei 225 and S\&P 500 futures are stock index futures, the Nikkei 225 is a price-weighted index, whereas the S\&P 500 is a value-weighted stock index. Therefore, we expect them to behave differently. 
The article is divided into five sections. The second section is devoted to a literature review. In the third section, the proposed M-GSV hedge ratio is discussed. The optimal hedge ratio is empirically estimated with the S\&P 500 futures and is compared with many different hedge ratios in the fourth section. The last section provides a summary and concluding comments.

\section{EXISTING HEDGE RATIOS}

In this section, we briefly review different hedge ratios that have been proposed. To facilitate a systematic discussion of these hedge ratios, we divide them into two broad categories: (a) risk-minimizing hedge ratios and (b) mean-risk hedge ratios. A list of the hedge ratios is presented in Table I for easy reference.

\section{Risk-Minimizing Hedge Ratio}

The basic concept of hedging is to combine spots and futures positions to form a hedged portfolio that will eliminate (or reduce) fluctuations in the value of the hedged portfolio. Specifically, consider a hedged portfolio consisting of one unit of spot position and $h$ units of futures position. Let $S_{t}$ and $F_{t}$ denote the spot and futures prices, respectively. Then, the profit on the so-called hedged portfolio, $R_{h}$, is given by

$$
R_{h}=R_{s}+h R_{f}
$$

\section{TABLE I}

List of Different Hedge Ratios

\begin{tabular}{llc}
\hline \multirow{2}{*}{ Notation } & \multicolumn{2}{c}{ Risk-Minimizing Hedge Ratio } \\
\cline { 2 - 3 } MV & \multicolumn{1}{c}{ Risk Measure } & Objective Function \\
MEG & Variance & Minimize (variance) risk \\
GSV & Mean-extended-Gini coefficient (MEG) & Minimize (MEG) risk \\
& Generalized semivariance (GSV) & Minimize (GSV) risk \\
Sharpe & \multicolumn{1}{c}{ Mean-Risk Hedge Ratio } \\
HKL & Standard deviation & Maximize Sharpe ratio \\
M-MEG & Variance & Maximize risk-return function \\
M-GSV & Mean-extended-Gini-coefficient & Maximize risk-return function \\
& Generalized semivariance (GSV) & Maximize risk-return function \\
\hline
\end{tabular}


where $R_{s}=S_{t+1}-S_{t}$ and $R_{f}=F_{t+1}-F_{t}$ are one-period profits on spot and futures, respectively. ${ }^{2}$ The standard risk-minimizing hedge ratio, which is known as the $M V$ hedge ratio, is obtained by minimization of the variance of $R_{h}$ (see Ederington, 1979; Johnson, 1960). Another riskminimizing hedge ratio, which is called the mean-extended-Gini (MEG) hedge ratio, is obtained by minimization of the MEG coefficient, $\Gamma_{v}\left(R_{h}\right)$, defined as follows (see Cheung et al., 1990; Kolb \& Okunev, 1992; Lien \& Luo, 1993; Lien \& Shaffer, 1999; Gregory-Allen \& Shalit, 1995):

$$
\Gamma_{v}\left(R_{h}\right)=-v \operatorname{Cov}\left(R_{h},\left(1-G\left(R_{h}\right)\right)^{v-1}\right)
$$

where $G$ is the cumulative probability distribution function and $v$ is the risk-aversion parameter. Note that $0 \leq v<1$ implies risk seekers, $v=1$ means risk-neutral, and $v>1$ means risk-averse investors. This MEG hedge ratio can be shown to be consistent with second-order stochastic dominance (see Shalit \& Yitzhaki, 1984, 1989; Yitzhaki, 1982, 1983). Furthermore, Shalit (1995) showed that if the prices are jointly normally distributed, the MEG hedge ratio will be the same as the MV hedge ratio. Yet another risk-minimizing hedge ratio is obtained by minimization of the following GSV (see De Jong et al., 1997; Lien \& Tse, 1998, 2000): ${ }^{3}$

$$
V_{\delta, \alpha}\left(R_{h}\right)=\int_{-\infty}^{\delta}\left(\delta-R_{h}\right)^{\alpha} d G\left(R_{h}\right), \quad \alpha>0
$$

where $G\left(R_{h}\right)$ is the probability distribution function of the return on the hedge portfolio $R_{h}$. This hedge ratio is called the GSV hedge ratio. The parameters $\delta$ and $\alpha$ (which are both real numbers) represent the target return and risk aversion, respectively. Here, the risk is defined in such a way that the investors consider possibilities of the returns below the target return $(\delta)$ as risky but not the returns above the target return. It can be shown (see Fishburn, 1977) that $\alpha<1$ represents a risk-seeking investor and $\alpha>1$ represents a risk-averse investor.

The GSV is consistent with the risk perceived by managers (see Crum et al., 1981; Lien \& Tse, 2000). Furthermore, as shown by

\footnotetext{
${ }^{2}$ Sometimes, the return on the hedged portfolio is considered, in which case $R_{s}=\left(S_{t+1}-S_{t}\right) / S_{t}$, $R_{f}=\left(F_{t+1}-F_{t}\right) / F_{t}$, and the hedge ratio is given by $h F_{t} / S_{t}$.

${ }^{3}$ De Jong et al. (1997) and Lien and Tse $(1998,2000)$ computed the optimal hedge ratios by minimizing the GSV given by Equation 3 for various values of $\alpha$. If the values of $\alpha$ are restricted to positive integers, GSV is also known as the lower partial moment. Because we allow $\alpha$ to be any positive real number, we prefer to call it the GSV instead of lower partial moments. Although both Fishburn (1977) and Bawa (1978) discussed the relationships among GSV, stochastic dominance, and expected utility, Bawa restricted the value of $\alpha$ to positive integers, whereas Fishburn allowed $\alpha$ to be any positive real value.
} 
Fishburn (1977) and Bawa (1975, 1978), the GSV is consistent with the concepts of stochastic dominance. Specifically, $V_{\delta, \alpha}\left(R_{h}\right)$ is consistent with the first-order (FSD), second-order (SSD), and third-order stochastic dominance (TSD) relations for $\alpha=0, \alpha=1$, and $\alpha=2$, respectively. ${ }^{4}$ Lien and Tse (1998) showed that if the futures and spot returns are jointly normally distributed and if the futures price follows a pure martingale process, the GSV hedge ratio will be equal to the MV hedge ratio.

\section{Mean-Risk Hedge Ratio}

All hedge ratios, discussed under the risk-minimizing hedge ratios, ignore the expected return aspect of the hedged portfolio. Next, we consider the class of hedge ratios that incorporate both risk and expected return in the derivation of the hedge ratios. These hedge ratios are called mean-risk hedge ratios. One example of a mean-risk hedge ratio is the Sharpe ratio-based hedge ratio considered by Howard and D'Antonio (1984). This hedge ratio, which is called the Sharpe hedge ratio, is obtained by maximization of the following risk-return function:

$$
\theta=\frac{E\left(R_{h}\right)-i}{\sigma_{h}}
$$

where $i$ is the risk-free rate. The Sharpe hedge ratio does not explicitly incorporate the risk-aversion parameter. A mean-risk hedge ratio that explicitly incorporates the risk-aversion parameter was considered by Hsin et al. (1994); the hedge ratio is obtained by maximization of the following risk-return function:

$$
V\left(E\left(R_{h}\right), \sigma_{h} ; A\right)=E\left(R_{h}\right)-0.5 A \sigma_{h}^{2}
$$

where $\sigma_{h}^{2}$ is equal to $\operatorname{Var}\left(R_{h}\right)$ and $A$ represents the risk-aversion parameter. This hedge ratio is called the HKL hedge ratio. Both the Sharpe and HKL hedge ratios will be the same as the MV hedge ratio if the futures price follows a pure martingale process.

However, another type of mean-risk hedge ratio is obtained by maximization of the following risk-return function (see Kolb \& Okunev, 1993):

$$
U\left(R_{h}\right)=E\left(R_{h}\right)-\Gamma_{v}\left(R_{h}\right)
$$

${ }^{4}$ We discuss the consistency of the GSV with the concept of stochastic dominance more when we come to the discussion of the proposed M-GSV hedge ratio. 
This risk-return function uses the MEG coefficient as the measure of risk. This hedge ratio is called the M-MEG hedge ratio. It can be shown that the M-MEG hedge ratio is consistent with SSD (see Shalit \& Yitzhaki, 1984, 1989; Yitzhaki, 1982, 1983). It is clear that if the futures price follows a pure martingale process, the M-MEG hedge ratio will be the same as the MEG hedge ratio. From Shalit (1995), it is clear that in addition to the martingale property, if futures and spot prices are jointly normally distributed, the M-MEG hedge ratio will be the same as the MV hedge ratio.

Kolb and Okunev (1992) found that for low levels of the riskaversion parameter $(v=2-5)$, the MEG hedge ratio is close to the MV hedge ratio, whereas for higher levels of the risk-aversion parameter, the MEG hedge ratio is quite different from the MV hedge ratio. However, Kolb and Okunev (1993) found that for higher levels of the risk-aversion parameter, the M-MEG hedge ratio converges to the MV hedge ratio.

\section{M-GSV HEDGE RATIO}

In this article, we extend the GSV hedge ratio considered by De Jong et al. (1997) and Lien and Tse $(1998,2000)$ to the M-GSV hedge ratio, where the optimal hedge ratio is obtained by maximization of the following risk-return function:

$$
U\left(R_{h}\right)=E\left[R_{h}\right]-V_{\delta, \alpha}\left(R_{h}\right)
$$

The M-GSV hedge ratio is similar to the GSV hedge ratio (Equation 3), except that the GSV hedge ratio ignores the expected return on the hedged portfolio. In this model, we do not use the risk-aversion parameter to multiply the GSV as done in the HKL model (see Equation 5). This is because the risk-aversion parameter is implicitly included in the definition of $V_{\delta, \alpha}\left(R_{h}\right)$, where $\alpha$ represents the risk aversion.

At this point, we explain the reason for extending the GSV hedge ratio to the M-GSV hedge ratio. This has to do with the fact that for the GSV hedge ratio to stochastically dominate all other hedge ratios, the GSV hedge ratio should be independent of the target return $\delta$ (see Bawa, 1975, 1978; Fishburn, 1977). It can be shown that, in general, the GSV hedge ratio is not independent of the target return. ${ }^{5}$ Therefore, a better way of using the GSV as a measure of risk is to base the hedge ratio on the mean-semivariance rule or the risk-return model developed by Fishburn (1977) and Bawa (1975, 1978), who provided the theoretical

${ }^{5}$ This issue is briefly discussed in the Appendix. 
justifications for such a mean-semivariance rule. This is accomplished by the proposed M-GSV hedge ratio, which is consistent with the risk-return model developed by Fishburn (1977) and Bawa (1975, 1978).

The GSV hedge ratio will be the same as the M-GSV hedge ratio if the futures price follows a pure martingale process. Furthermore, as shown by Lien and Tse (1998), if the futures price follows a pure martingale process and if the futures and spot returns are jointly normal, the MV hedge ratio will be the same as the GSV hedge ratio. Because the M-GSV and GSV hedge ratios are the same under the martingale property, M-GSV, GSV, and MV hedge ratios will be the same if joint normality holds in addition to the martingale property. Another condition, which does not require the normality assumption, is given in the following proposition:

Suppose that the futures price follows a pure martingale process (i.e., the return on the futures is equal to zero). Furthermore, suppose that the return on the hedged portfolio is symmetrically distributed around the target return $\delta$. Then, the M-GSV, GSV, and MV hedge ratios will be the same for $\alpha=2$.

As for the estimation of the M-GSV hedge ratio, there are different methods that can be used (see Ahmadi, Sharp, \& Walther, 1986; Lien \& Tse, 1998, 2000). We choose the empirical distribution-based technique because of its simplicity and desirable small-sample property. For example, the estimate of GSV based on the empirical distribution is unbiased (see Ahmadi et al., 1986).

\section{EMPIRICAL ANALYSIS}

We estimate the M-GSV hedge ratio and compare it with five other hedge ratios (see Table I) with the weekly S\&P 500 futures data from April 21, 1982 to December 27, 1991 (490 weeks of data). Data were obtained from Datastream.

Before estimating any of the hedge ratios, we must determine whether the futures and spot price changes are normally distributed and whether the futures price follows a pure martingale process. The normality and pure martingale tests on the S\&P 500 futures are given in Table II. It is clear that the expected futures price change is positive and is more than eight times its standard error. This clearly indicates that the futures price does not follow a pure martingale process. With respect to normality, we computed two different statistics. The Jarque-Bera test statistics (see Jarque \& Bera, 1987) are highly significant (even at the 
TABLE II

Summary Statistics of the Spot and Futures Price Change

\begin{tabular}{lcc}
\hline Statistics & Spot Price Change & Futures Price Change \\
\hline Sample mean & 0.5934 & 0.5928 \\
Standard error of mean (SE) & 0.0727 & 0.0730 \\
Skewness & -3.546 & -5.023 \\
Standardized kurtosis & 41.200 & 68.356 \\
Jarque-Bera test & $35,683.09$ & $97,456.85$ \\
D'Agostino D statistic & -32.908 & -41.879 \\
Number of observations & 490 & 490 \\
\hline
\end{tabular}

Note: The standard error of the sample mean is computed with the following formula, which corrects for the serial autocorrelation:

$$
\mathrm{SE}=\sqrt{\frac{1}{T} \sum_{j=-(\mathrm{T}-1)}^{j=\mathrm{T}-1}\left[1-\frac{|j|}{T}\right] \gamma(j)}
$$

where $\gamma(j)$ denotes the lag $j$ autocovariance. The Jarque-Bera test statistic is based on Jarque and Bera (1987) and is asymptotically chi-square-distributed with 2 degrees of freedom. The critical values at 5 and $1 \%$ are 5.991 and 9.210 , respectively. The D'Agostino $D$ statistic is based on D'Agostino (1971) and is asymptotically distributed as a normal $N(0,1)$. The critical values at 5 and $1 \%$ are 1.960 and 2.576 , respectively.

$0.5 \%$ level) for both spot and futures price changes. The same conclusion is true for the D'Agostino D statistic (D'Agostino, 1971). Therefore, it is clear that neither normality nor the pure martingale hypothesis hold for S\&P 500 spot and futures prices. This means that for the S\&P 500 futures, Sharpe, HKL, GSV, M-GSV, MEG, and M-MEG ratios are expected to be different from the standard MV hedge ratio. Because one of the objectives of the article is to compare the M-GSV hedge ratio with other hedge ratios, we first discuss the estimation of the MV, Sharpe, and HKL hedge ratios. The MV hedge ratio is equal to -0.8523 . As for the Sharpe hedge ratio, in our case it does not exist. This has to do with the fact that the Sharpe ratio given by Equation 4 continuously increases with the hedge ratio. Therefore, there is no finite optimal solution. Actually, if we use the formula given by Howard and D'Antonio (1984, their Equation 6), we get the hedge ratio corresponding to the minimum, instead of the maximum, Sharpe ratio. This is because the second-order condition is not satisfied. For our sample, the dependence of the Sharpe ratio on the hedge ratio is shown in Figure 1. From the figure, it can be seen that the Sharpe ratio does not have the maximum, and the zero slope point corresponds to the minimum Sharpe ratio instead of the maximum one. Such behavior makes the Sharpe ratio-based hedge ratio less reliable. This is also supported by the fact that De Jong et al. (1997) found the hedge ratio fluctuated widely (e.g., from -41.41 to 88.48 for Japanese yen). 


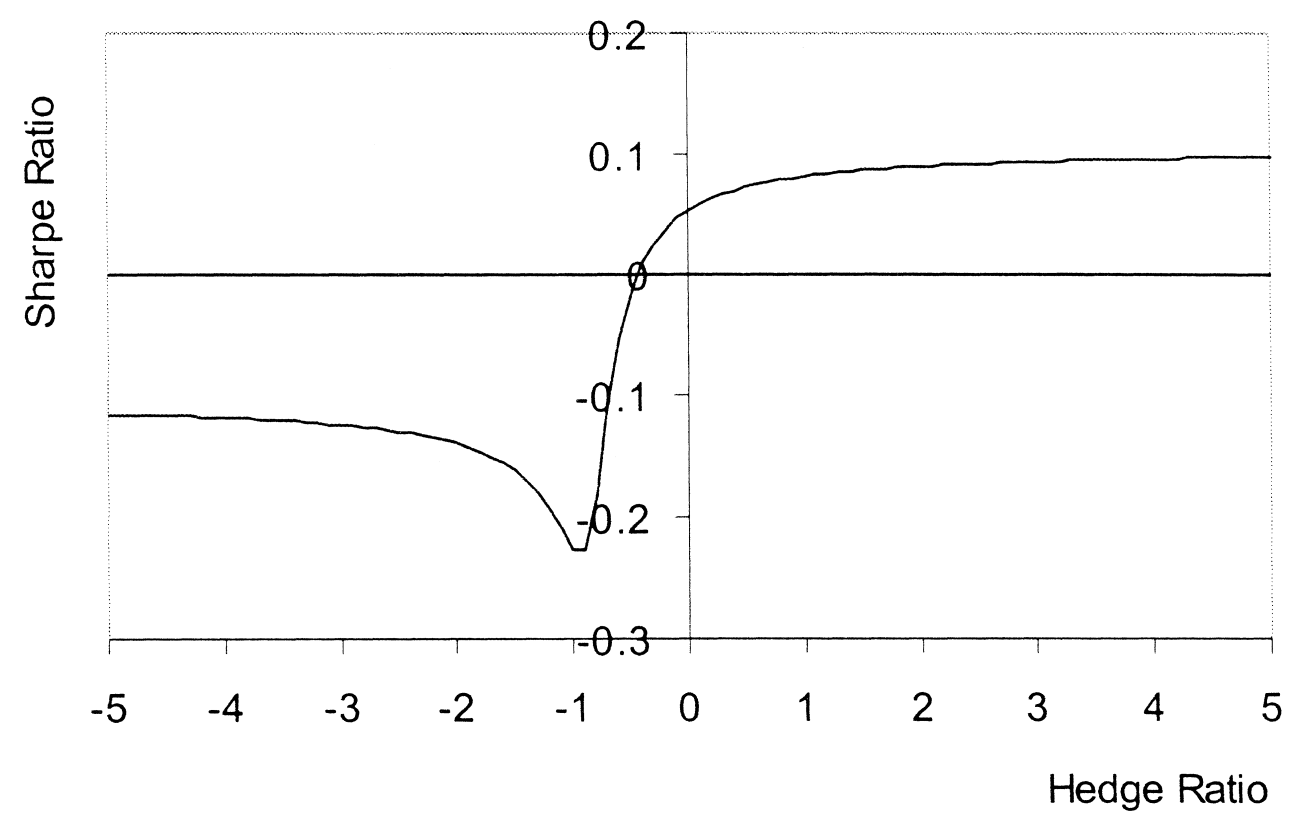

FIGURE 1

Sharpe ratio as a function of the hedge ratio (Howard-D'Antonio model).

Risk Aversion (A)

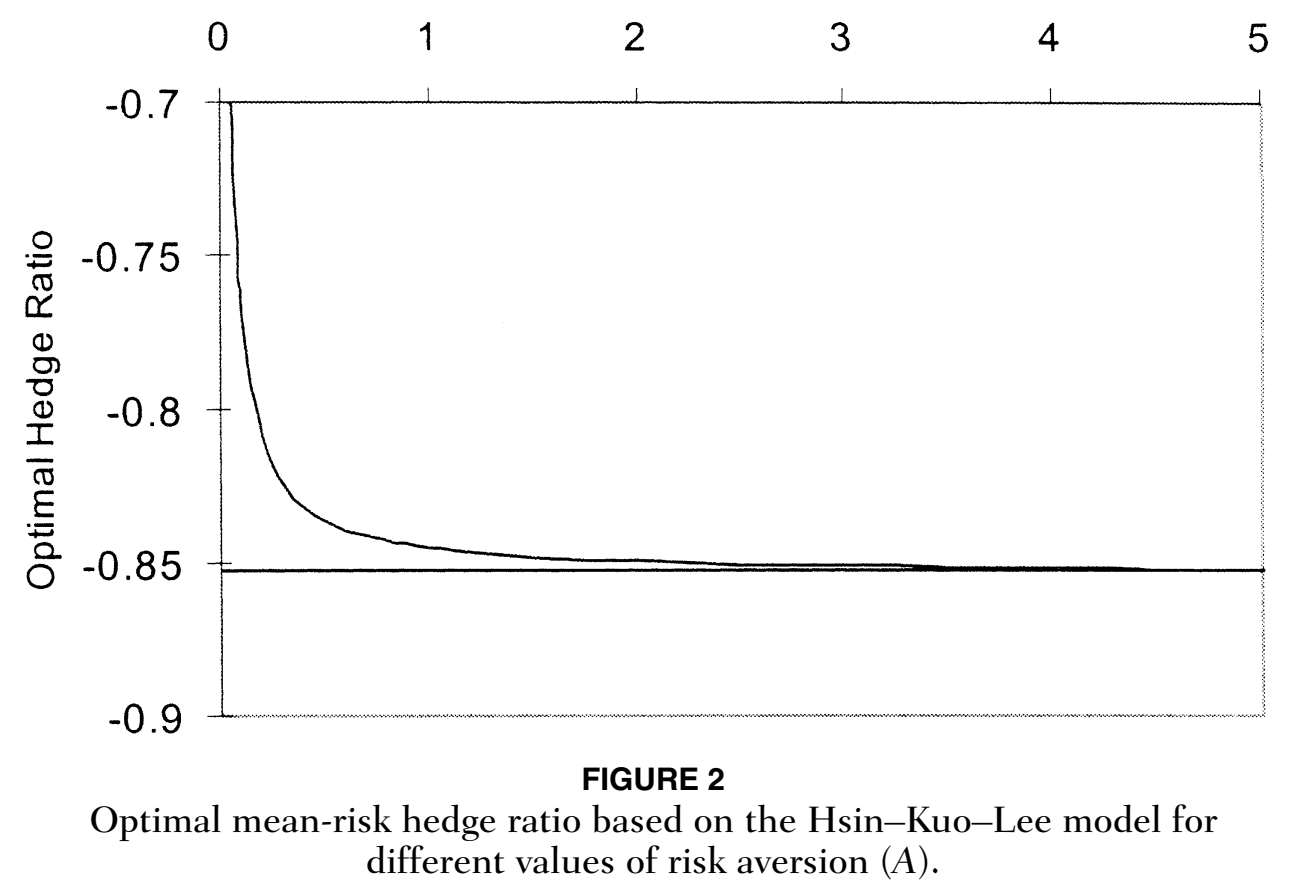

Next, we estimate different mean-risk (HKL) hedge ratios based on the Hsin-Kuo-Lee model (Equation 5) for different values of the riskaversion parameter $(A)$. The optimal HKL hedge ratios are shown in Figure 2. It is clear from the diagram that the optimal HKL hedge ratio smoothly converges to the MV hedge ratio for values of $A$ greater than 3.0. This is expected because for high values of the risk-aversion parameter, the expected return becomes less important and the resulting hedge ratio will be the same as the risk-minimizing hedge ratio. 
Now we turn to the estimation of the MEG- and GSV-based hedge ratios. Before the GSV-based hedge ratios can be estimated, we need to decide on the two parameters: the target return $\delta$ and the risk-aversion parameter $\alpha$. In the empirical analysis, two different target returns $\delta$ are used. First, the target return $\delta$ is set to the sample average of the S\&P 500 spot price changes (which is equal to 0.5933 per week). This choice is consistent with the empirical result obtained by Harlow and Rao (1989). They inferred that the target return is related to the market mean return. Alternatively, the target return $\delta$ is set to zero (this is the value used by De Jong et al., 1997). Once the value of $\delta$ is fixed, the optimal hedge ratios are calculated for a range of values of the riskaversion parameter $\alpha$ (1.25-60). Because the MEG-based hedge ratios also depend on the risk-aversion parameter $(v)$, these hedge ratios are also estimated for the same range of values for the risk-aversion parameter $v$. However, even if we use the same range for $\alpha$ and $v$, a value of $\alpha$ does not correspond to the same value of $v$; that is, $v=1$ is not equivalent to $\alpha=1 .^{6}$

The estimates of the MEG and GSV hedge ratios are presented in Table III and plotted in Figure 3. The GSV hedge ratio is higher than the MEG hedge ratio. Both the GSV and MEG hedge ratios seem to converge to fixed but different values as the risk-aversion parameter becomes large. The MEG hedge ratio seems to converge to a ratio that is close to the MV hedge ratio. However, the GSV hedge ratio seems to converge to a higher value. We discuss this issue of convergence more when we discuss the M-MEG and M-GSV hedge ratios next.

The remaining two hedge ratios, M-GSV and M-MEG hedge ratios, are presented in Table IV for different values of the risk-aversion parameters $\alpha$ and $v$. Figure 4 plots the two hedge ratios as well as the MV hedge ratio $(-0.8523)$. As before, two different values of the target return $\left[\delta=0\right.$ and $\left.\delta=E\left(R_{s}\right)\right]$ are used. First, we compare the estimates of M-GSV and GSV hedge ratios. As discussed earlier, for the GSV-based models the relevant range of $\alpha$ is $\alpha \leq 2$ (see Footnote 6). For this range, the M-GSV hedge ratio varies less $\left[-0.8562\right.$ to -0.8652 for $\left.\delta=E\left(R_{s}\right)\right]$ than the GSV hedge ratio, which varies from -0.8699 to -0.8967 . Furthermore, the M-GSV leads to less hedging than the GSV. This is reasonable because the sample mean of the futures price changes is

\footnotetext{
${ }^{6}$ Because some of the most widely used assumptions regarding utility functions are either risk aversion or risk aversion with decreasing absolute risk aversion, the most relevant range of values for $\alpha$ is $\alpha \leq 2$. This range also covers the FSD, SSD, and TSD relations. However, we estimated the hedge ratios for values of $\alpha$ greater than 2 to see if there was any indication of the hedge ratios converging to a fixed value and to see if there was any association between the higher values of $\alpha$ and higher values of $v$ for the MEG hedge ratio.
} 


\section{TABLE III}

Risk-Minimizing Generalized-Semivariance (GSV) and Mean-Extended-Gini Coefficient (MEG) Hedge Ratios for Different Values of Risk-Aversion Parameters

\begin{tabular}{|c|c|c|c|c|}
\hline \multicolumn{3}{|c|}{ GSV Hedge Ratio } & \multicolumn{2}{|c|}{ MEG Hedge Ratio } \\
\hline$\alpha$ & $\delta=E\left(R_{s}\right)$ & $\delta=0$ & $v$ & Hedge Ratio \\
\hline 1.25 & -0.8967 & -0.9013 & 1.25 & -0.9045 \\
\hline 1.50 & -0.8913 & -0.8879 & 1.50 & -0.9171 \\
\hline 1.75 & -0.8816 & -0.8739 & 1.75 & -0.9206 \\
\hline 2.00 & -0.8699 & -0.8603 & 2.00 & -0.9222 \\
\hline 2.25 & -0.8567 & -0.8452 & 2.25 & -0.9227 \\
\hline 2.50 & -0.8424 & -0.8292 & 2.50 & -0.9227 \\
\hline 2.75 & -0.8271 & -0.8126 & 2.75 & -0.9224 \\
\hline 3.00 & -0.8107 & -0.7951 & 3.00 & -0.9218 \\
\hline 4.00 & -0.7484 & -0.7377 & 4.00 & -0.9186 \\
\hline 5.00 & -0.7210 & -0.7158 & 5.00 & -0.9138 \\
\hline 6.00 & -0.7083 & -0.7051 & 6.00 & -0.9089 \\
\hline 7.00 & -0.7011 & -0.6989 & 7.00 & -0.9047 \\
\hline 8.00 & -0.6966 & -0.6949 & 8.00 & -0.9005 \\
\hline 9.00 & -0.6934 & -0.6921 & 9.00 & -0.8968 \\
\hline 10.00 & -0.6911 & -0.6900 & 10.00 & -0.8933 \\
\hline 20.00 & -0.6822 & -0.6818 & 20.00 & -0.8713 \\
\hline 30.00 & -0.6797 & -0.6794 & 30.00 & -0.8570 \\
\hline 40.00 & -0.6784 & -0.6782 & 40.00 & -0.8510 \\
\hline 50.00 & -0.6777 & -0.6775 & 50.00 & -0.8476 \\
\hline 60.00 & -0.6772 & -0.6771 & 60.00 & -0.8476 \\
\hline \multicolumn{4}{|c|}{ Minimum-variance (Johnson) hedge ratio } & -0.8523 \\
\hline
\end{tabular}

positive (0.5928; see Table II). Therefore, a more negative hedge ratio means a lower expected profit on the hedged portfolio. Because the M-GSV incorporates both expected profit and risk, the M-GSV hedge ratio should lead to less hedging (i.e., a less negative hedge ratio) than the GSV hedge ratio.

Another interesting observation is that both the M-GSV and MMEG hedge ratios are found to converge to fixed but different values as the risk-aversion parameter becomes large. The M-MEG hedge ratio seems to converge to a value that is close to the MV hedge ratio. However, the M-GSV hedge ratio seems to converge to a higher value; that is, the M-MEG hedge ratio will lead to overhedging compared to the M-GSV hedge ratio. The convergence of the M-MEG and M-GSV hedge ratios needs to be discussed further. Our result, which indicates the convergence of the M-MEG hedge ratio to the MV hedge ratio, is not universal. As mentioned before, Kolb and Okunev (1992) found the MEG hedge ratios to converge to values different than those of the MV hedge ratios for five different commodities (including the S\&P 500 


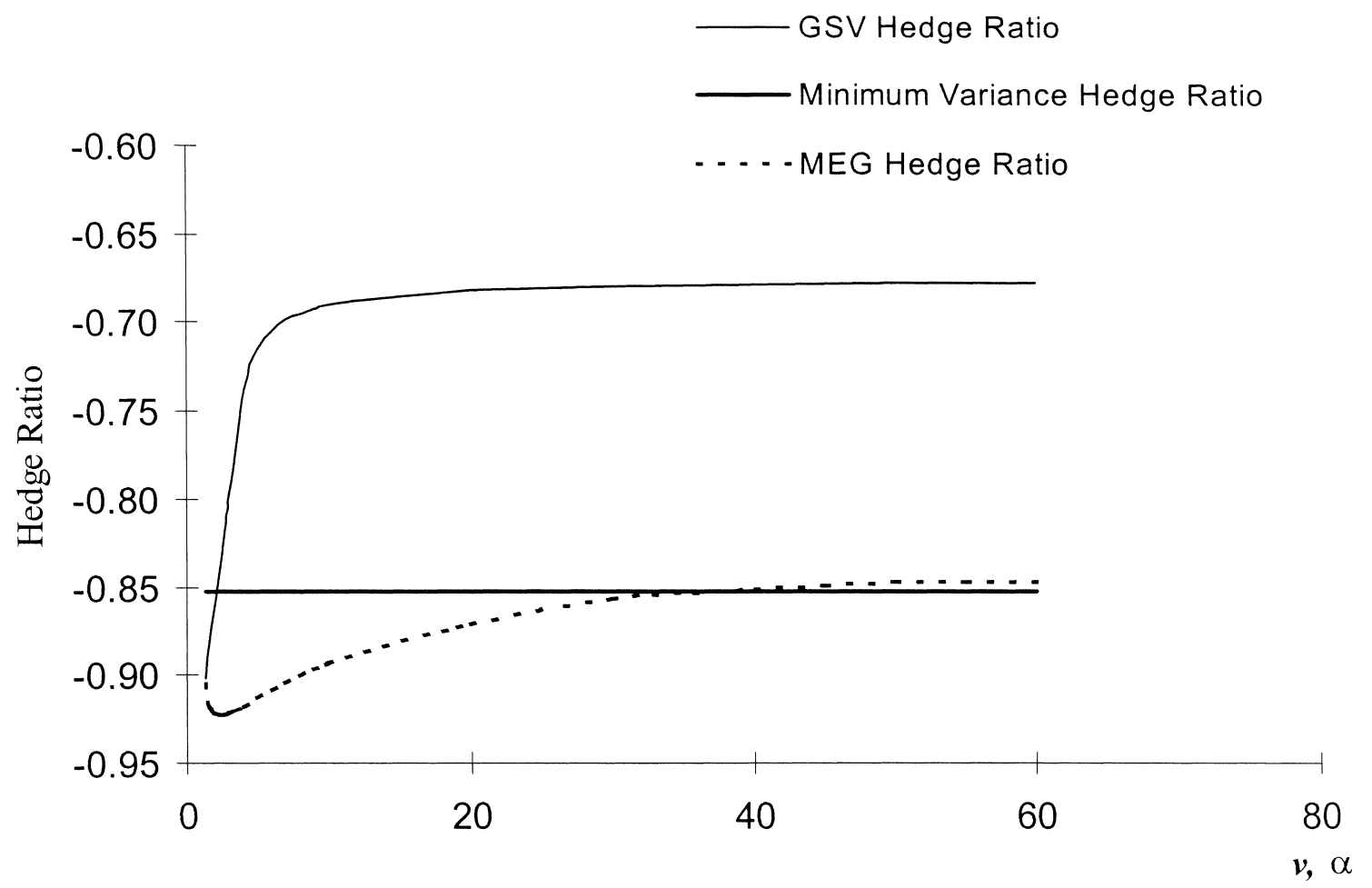

FIGURE 3

Risk-minimizing GSV and MEG hedge ratios for different values of risk-aversion parameters. The GSV hedge ratio is calculated with $\delta=E\left(R_{s}\right)$. The values for the GSV hedge ratio are taken from the second column of Table III. Similarly, the values for the MEG coefficient hedge ratio are taken from the fifth column of Table III. The MV hedge ratio is equal to -0.8523 .

futures). ${ }^{7}$ However, Kolb and Okunev (1993) found that the M-MEG hedge ratio converges close to the MV hedge ratio.

As for the convergence of the M-GSV hedge ratio, the finding that the M-GSV hedge ratio converges to a value different from the MV hedge ratio is not surprising. The M-GSV hedge ratio is expected to be different from the MV hedge ratio because the M-GSV hedge ratio uses the GSV to measure the risk, whereas the MV hedge ratio uses variance as the measure of risk. So long as these two measures of risk are different, the two hedge ratios are expected to be different. Because the GSV considers below target return to be risky whereas variance considers both above and below mean return to be risky, these two risk measures are expected to be different unless the joint normality condition or the symmetric distribution condition (as given in the proposition) is satisfied. From Table II, it is clear that none of these conditions is satisfied. Therefore, we expect the M-GSV hedge ratio to converge to values different from the MV hedge ratio.

${ }^{7}$ They consider daily data for corn, copper, German mark, gold, and S\&P 500 futures for 1989. 


\section{TABLE IV}

M-GSV Hedge Ratio and M-MEG Hedge Ratios for Different Values of Risk-Aversion Parameters

\begin{tabular}{|c|c|c|c|c|}
\hline \multicolumn{3}{|c|}{ M-GSV Hedge Ratio } & \multicolumn{2}{|c|}{ M-MEG Hedge Ratio } \\
\hline$\alpha$ & $\delta=E\left(R_{s}\right)$ & $\delta=0$ & $v$ & Hedge Ratio \\
\hline 1.25 & -0.8562 & -0.8562 & 1.25 & -0.7955 \\
\hline 1.50 & -0.8652 & -0.8582 & 1.50 & -0.8600 \\
\hline 1.75 & -0.8647 & -0.8548 & 1.75 & -0.8800 \\
\hline 2.00 & -0.8591 & -0.8474 & 2.00 & -0.8887 \\
\hline 2.25 & -0.8500 & -0.8368 & 2.25 & -0.8930 \\
\hline 2.50 & -0.8383 & -0.8241 & 2.50 & -0.8957 \\
\hline 2.75 & -0.8246 & -0.8095 & 2.75 & -0.8972 \\
\hline 3.00 & -0.8092 & -0.7933 & 3.00 & -0.8978 \\
\hline 4.00 & -0.7483 & -0.7376 & 4.00 & -0.8976 \\
\hline 5.00 & -0.7210 & -0.7158 & 5.00 & -0.8953 \\
\hline 6.00 & -0.7083 & -0.7051 & 6.00 & -0.8921 \\
\hline 7.00 & -0.7011 & -0.6989 & 7.00 & -0.8894 \\
\hline 8.00 & -0.6966 & -0.6949 & 8.00 & -0.8869 \\
\hline 9.00 & -0.6934 & -0.6921 & 9.00 & -0.8848 \\
\hline 10.00 & -0.6911 & -0.6900 & 10.00 & -0.8813 \\
\hline 20.00 & -0.6822 & -0.6818 & 20.00 & -0.8615 \\
\hline 30.00 & -0.6797 & -0.6794 & 30.00 & -0.8512 \\
\hline 40.00 & -0.6784 & -0.6782 & 40.00 & -0.8476 \\
\hline 50.00 & -0.6777 & -0.6775 & 50.00 & -0.8462 \\
\hline 60.00 & -0.6772 & -0.6771 & 60.00 & -0.8462 \\
\hline \multicolumn{3}{|c|}{ Minimum-variance (Johnson) hedge ratio } & & -0.8523 \\
\hline
\end{tabular}

Note: $\quad \mathrm{M}-\mathrm{GSV}=$ mean-generalized-semivariance; $\mathrm{M}-\mathrm{MEG}=$ mean-extended-Gini.

\section{SUMMARY AND CONCLUSION}

In this article, we extended the GSV-minimizing hedge ratio considered by De Jong et al. (1997) and Lien and Tse $(1998,2000)$ to an M-GSV hedge ratio. This article differs from the existing work, especially by De Jong et al. and Lien and Tse (1998, 2000), in many respects. First, we extended the risk-minimizing hedge ratio to a risk-return hedge ratio. This makes the M-GSV hedge ratio consistent with the risk-return model developed by Fishburn (1977) and Bawa (1975, 1978). Second, we clarified an issue regarding the consistency of the GSV hedge ratio with respect to stochastic dominance. We showed that the GSV hedge ratio, considered by De Jong et al. and Lien and Tse (1998, 2000), is not consistent with stochastic dominance. Third, we showed that under some circumstances the assumption of symmetrical distribution is sufficient to have the M-GSV hedge ratio converge to the standard MV hedge ratio. 


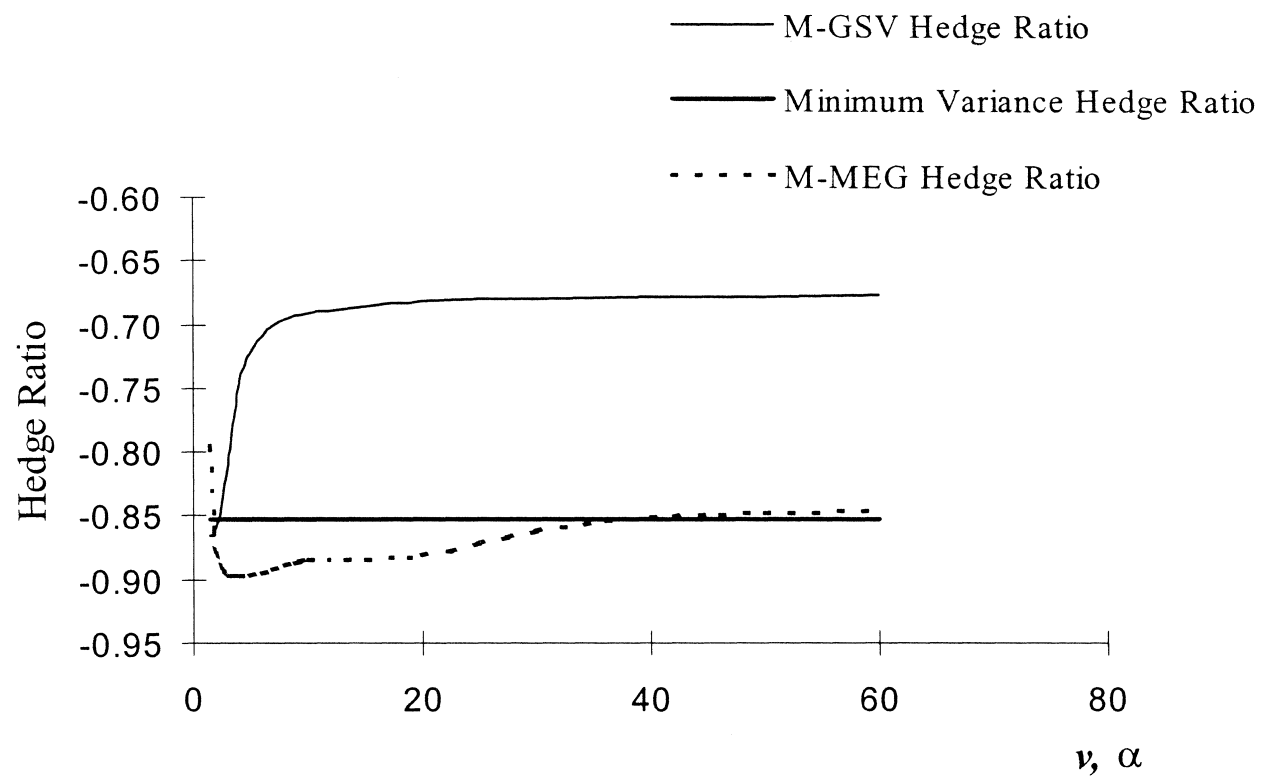

FIGURE 4

M-GSV hedge ratio and M-MEG hedge ratio for different values of riskaversion parameters. The M-GSV hedge ratio is calculated with $\delta=E\left(R_{\mathrm{s}}\right)$. The values for the GSV hedge ratio are taken from the second column of Table IV. Similarly, the values for the M-MEG hedge ratio are taken from the fifth column of Table IV. The MV hedge ratio is equal to -0.8523 .

Fourth, we compared the M-GSV hedge ratio to other hedge ratios, which include the MEG coefficient (M-MEG) hedge ratio, the Sharpe ratiobased hedge ratio, the standard mean-variance hedge ratio, and the MV hedge ratio. Fifth, we estimated all seven hedge ratios for the S\&P 500 futures, which is different from the currency futures used by De Jong et al. and the Nikkei 225 futures analyzed by Lien and Tse (1998, 2000). Finally, we performed two different types of normality tests (Jarque-Bera and D'Agostino tests) as well as the martingale hypothesis tests to see if different hedge ratios converge to the MV hedge ratio.

We found that the S\&P 500 futures and spot prices are neither jointly nor individually normally distributed. Furthermore, the martingale hypothesis does not hold, indicating that the expected futures price change is not zero. Therefore, we conclude that the various hedge ratios considered are different from the standard MV hedge ratio.

We found that the M-GSV hedge ratio varies less than the GSV hedge ratio for lower and relevant levels of risk aversion. The M-GSV hedge ratio converges to a value higher than the MV hedge ratio for large values of risk aversion. However, the M-MEG hedge ratio converges to a value close to the MV hedge ratio for large values of the risk aversion. This can be explained by the fact that the M-GSV hedge ratio uses GSV as the risk measure, unlike the MV hedge ratio, which uses variance as the risk measure. 


\section{APPENDIX}

According to the analyses of Fishburn (1977) and Bawa (1975, 1978), for the GSV hedge ratio to stochastically dominate all other hedge ratios, the GSV hedge ratio should have the minimum GSV for all values of the target return $\delta$. This condition will be satisfied if the GSV hedge ratio is independent of the target return $(\delta)$. It can be shown that the derivative of the GSV hedge ratio $h^{*}$ with respect to $\delta$ is given by the following (see Lien \& Tse, 1998, 2000):

$$
\frac{d h^{*}}{d \delta}=-\frac{\int_{-\infty}^{+\infty} \int_{-\infty}^{\delta-h R_{f}}\left(\delta-R_{s}-h R_{f}\right)^{\alpha-2} R_{f} f\left(R_{s}, R_{f}\right) d R_{s} d R_{f}}{\int_{-\infty}^{+\infty} \int_{-\infty}^{\delta-h R_{f}}\left(\delta-R_{s}-h R_{f}\right)^{\alpha-2} R_{f}^{2} f\left(R_{s}, R_{f}\right) d R_{s} d R_{f}}
$$

where $f\left(R_{s}, R_{f}\right)$ is the joint density function. ${ }^{8}$ Therefore, for $\frac{d h^{*}}{d \delta}$ to be equal to zero, we need the following condition to be satisfied:

$$
\int_{-\infty}^{+\infty} \int_{-\infty}^{\delta-h R_{f}}\left(\delta-R_{s}-h R_{f}\right)^{\alpha-2} R_{f} f\left(R_{s}, R_{f}\right) d R_{s} d R_{f}=0
$$

In general, this condition is not satisfied. Therefore, in a strict sense the GSV hedge ratio will not be consistent with the concept of stochastic dominance relations and the expected utility model. ${ }^{9}$

\section{BIBLIOGRAPHY}

Ahmadi, H. Z., Sharp, P. A., \& Walther, C. H. (1986). The effectiveness of futures and options in hedging currency risk. Advances in Futures and Option Research, 1, 171-191.

Bawa, V. S. (1975). Optimal rules for ordering uncertain prospects. Journal of Financial Economics, 2, 95-121.

Bawa, V. S. (1978). Safety-first, stochastic dominance, and optimal portfolio choice. Journal of Financial and Quantitative Analysis, 13, 255-271.

Cecchetti, S. G., Cumby, R. E., \& Figlewski, S. (1988). Estimation of the optimal futures hedge. Review of Economics and Statistics, 70, 623-630.

Cheung, C. S., Kwan, C. C. Y., \& Yip, C. Y. (1990). The hedging effectiveness of options and futures: A mean-Gini approach. Journal of Futures Markets, $10,61-74$.

${ }^{8}$ Equation Al corresponds to Equation 9 in Lien and Tse (2000), except that the negative sign is missing in Lien and Tse (2000).

${ }^{9}$ Note, however, that if the return distributions are jointly normal and the futures price follows a pure martingale process, the GSV hedge ratio will be consistent with the concepts of stochastic dominance relations and the expected utility hypothesis. This is so because the optimal hedge ratio will be equal to the MV hedge ratio, which is independent of $\delta$. 
Crum, R. L., Laughhunn, D. L., \& Payne, J. W. (1981). Risk-seeking behavior and its implications for financial models. Financial Management, 10(5), 20-27.

D'Agostino, R. B. (1971). An omnibus test of normality for moderate and large size samples. Biometrika, 58, 341-348.

De Jong, A., De Roon, F., \& Veld, C. (1997). Out-of-sample hedging effectiveness of currency futures for alternative models and hedging strategies. Journal of Futures Markets, 17, 817-837.

Ederington, L. H. (1979). The hedging performance of the new futures markets. Journal of Finance, 34, 157-170.

Fishburn, P. C. (1977). Mean-risk analysis with risk associated with belowtarget returns. American Economic Review, 67(2), 116-126.

Gregory-Allen, R. B., \& Shalit, H. (1999). The estimation of systemic risk under differentiated risk-aversion: A mean-extended Gini approach. Review of Quantitative Finance and Accounting, 12, 135-157

Harlow, W. V., \& Rao, R. K. S. (1989). Asset pricing in a generalized mean-lower partial moment framework: Theory and evidence. Journal of Financial and Quantitative Analysis, 24, 285-311.

Howard, C. T., \& D’Antonio, L. J. (1984). A risk-return measure of hedging effectiveness. Journal of Financial and Quantitative Analysis, 19, 101-112.

Hsin, C. W., Kuo, J., \& Lee, C. F. (1994). A new measure to compare the hedging effectiveness of foreign currency futures versus options. Journal of Futures Markets, 14, 685-707.

Jarque, C. M., \& Bera, A. K. (1987). A test for normality of observations and regression residuals. International Statistical Review, 55, 163-172.

Johnson, L. L. (1960). The theory of hedging and speculation in commodity futures. Review of Economic Studies, 27, 139-151.

Kolb, R. W., \& Okunev, J. (1992). An empirical evaluation of the extended mean-Gini coefficient for futures hedging. Journal of Futures Markets, 12, 177-186.

Kolb, R. W., \& Okunev, J. (1993). Utility maximizing hedge ratios in the extended mean Gini framework. Journal of Futures Markets, 13, 597-609.

Lien, D., \& Luo, X. (1993). Estimating the extended mean-Gini coefficient for futures hedging. Journal of Futures Markets, 13, 665-676.

Lien, D., \& Shaffer, D. R. (1999). A note on estimating the minimum extended Gini hedge ratio. Journal of Futures Markets, 19, 101-113.

Lien, D., \& Tse, Y. K. (1998). Hedging time-varying downside risk. Journal of Futures Markets, 18, 705-722.

Lien, D., \& Tse, Y. K. (2000). Hedging downside risk with futures contracts. Applied Financial Economics, 10(2), 163-170.

Myers, R. J., \& Thompson, S. R. (1989). Generalized optimal hedge ratio estimation. American Journal of Agricultural Economics, 71, 858-868.

Shalit, H. (1995). Mean-Gini hedging in futures markets. Journal of Futures Markets, 15, 617-635.

Shalit, H., \& Yitzhaki, S. (1984). Mean-Gini, portfolio theory, and the pricing of risky assets. Journal of Finance, 39, 1449-1468. 
Shalit, H., \& Yitzhaki, S. (1989). Evaluating the mean-Gini approach to portfolio selection. International Journal of Finance, 1(2), 15-31.

Yitzhaki, S. (1982). Stochastic dominance, mean variance, and Gini's mean difference. American Economic Review, 72(1), 178-185.

Yitzhaki, S. (1983). On an extension of the Gini inequality index. International Economic Review, 24, 617-628. 